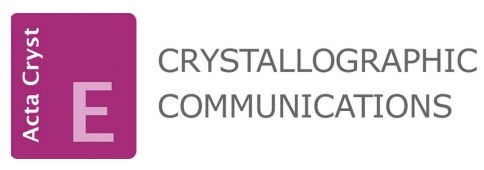

ISSN 2056-9890

Received 13 May 2020

Accepted 19 May 2020

Edited by A. V. Yatsenko, Moscow State University, Russia

Keywords: crystal structure; dimethoxybenzene; two-photon absorption; Hirshfeld surface; DFT calculations; absorption and emission spectra.

CCDC reference: 2004742

Supporting information: this article has supporting information at journals.iucr.org/e

\section{Molecular and crystal structure, optical properties and DFT studies of 1,4-dimethoxy-2,5-bis[2-(4- nitrophenyl)ethenyl]benzene}

\author{
Georgii Bogdanov, ${ }^{\mathrm{a}, \mathrm{b} *}$ Evgenii Oskolkov, ${ }^{\mathrm{a}}$ Jenna Bustos, ${ }^{\text {a }}$ Viktor Glebov, ${ }^{\mathrm{a}}$ John P. \\ Tillotson $^{\mathrm{c}}$ and Tatiana V. Timofeeva ${ }^{\mathrm{a}}$
}

aDepartment of Chemistry, New Mexico Highlands University, Las Vegas, New Mexico, 87701, USA, 'Department of
Chemical and Biomolecular Engineering, University of California Irvine, Irvine, California, 92617, USA, and ${ }^{\mathbf{c}}$ School of
Chemistry and Biochemistry, Georgia Institute of Technology, Atlanta, Georgia, 30332, USA. *Correspondence e-mail:
bogdgv@gmail.com

The title compound DBNB, $\mathrm{C}_{24} \mathrm{H}_{20} \mathrm{~N}_{2} \mathrm{O}_{6}$, has been crystallized and studied by $\mathrm{X}$-ray diffraction, spectroscopic and computational methods. In the title molecule, which is based on a 1,4-distyryl-2,5-dimethoxybenzene core with $p$-nitro-substituted terminal benzene rings, the dihedral angle between mean planes of the central fragment and the terminal phenyl ring is $16.46(6)^{\circ}$. The crystal packing is stabilized by $\pi-\pi$ interactions. DFT calculations at the B3LYP/ 6-311 G(d,p) level of theory were used to compare the optimized structures with the experimental data. Energy parameters, including HOMO and LUMO energies, their difference, and vertical excitation and emission energies were obtained.

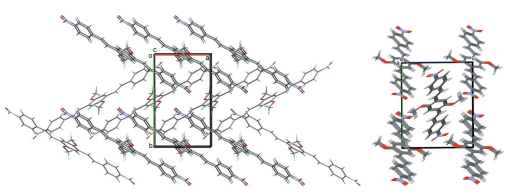

OPEN 2 ACCESS

\section{Chemical context}

One method for the design of the organic two-photon absorbing (TPA) molecules is Donor- $\pi$-Bridge-Acceptor $-\pi$ Bridge-Donor or Acceptor- $\pi$-bridge-Donor- $\pi$-bridgeAcceptor (He et al., 2008). Specific spectroscopic properties of such molecules make them useful for applications in different areas. For instance, about half a century ago it was found that the title compound and other substituted distyrylbenzenes would be highly efficient wavelength shifters in organic liquid scintillators (Nakaya et al., 1966). It is important to mention that some molecules with such general structure possess not only plasminogen activator (tPA) activity but also demonstrate light emission, which make them useful for organic light-emitting diodes (OLEDs) (Cárdenas et al., 2019) and/or chemical sensors (Xu et al., 2013). For instance, for a molecule similar to the title molecule, 1,4-dimethoxy-2,5-bis(4'-dichlorostyryl)benzene, blue fluorescence emission was found, which makes it a prospective candidate for cell imaging. Another phenyleneethenylene derivative, 2,5-dimethoxy-1,4bis[2-(4-carboxylatestyryl)]benzene, for which two polymorphs and one DMF solvate have been studied, demonstrated three different types of emission, depending on the molecular packing in the crystal (Cárdenas et al., 2019). On this basis, we considered that an investigation of the molecular structure and crystal packing of the title compound would be useful for correlating its structural characteristics to its spectroscopic properties. 


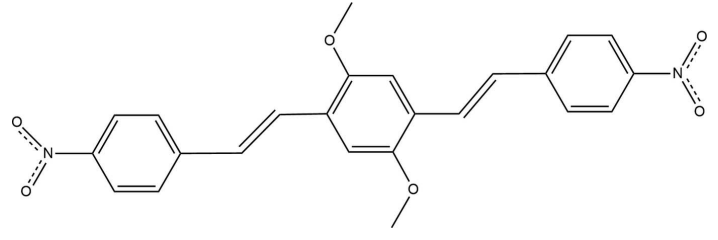

\section{Structural commentary}

The molecular structure of DBDB is presented in Fig. 1. The molecule lies on an inversion center and shows a slight deviation from planarity. The dihedral angle formed by mean planes of the central fragment and the terminal benzene ring is $16.46(6)^{\circ}$. The methoxy group is rotated by $3.77(11)^{\circ}$ and the nitro group by $15.99(8)^{\circ}$ with respect to the central ring and the terminal benzene ring, respectively. In a similar compound with para-chlorine substitution, the angles between the central and terminal aromatic rings are 43.82 and $67.38^{\circ}$ (Xu et al., 2013), whereas in closely related structures these angles vary from 11.97 to $35.75^{\circ}$ (Cárdenas et al., 2019), demonstrating the flexibility of this type of molecule, even in the solid state.

\section{Supramolecular features}

In the crystal, the DBDB molecules are packed into ladderlike stacks (Fig. 2) along the $a$-axis direction, which in turn build a parquet-like structure (Fig. 3). An intermolecular distance of 3.451 (1) $\AA$ is found between the mean planes of the central rings in the molecular stacks, with a separation between the centroids of the central ring and the terminal benzene ring of 3.899 (1) $\AA$, which suggests the presence of $\pi$ $\pi$ interactions between the molecules.

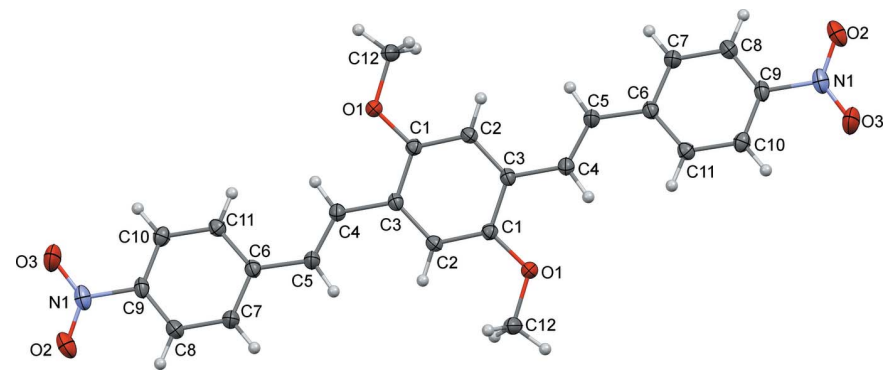

Figure 1

A view of the molecular structure of the title compound with the atomlabeling scheme. Displacement ellipsoids are drawn at the $50 \%$ probability level.

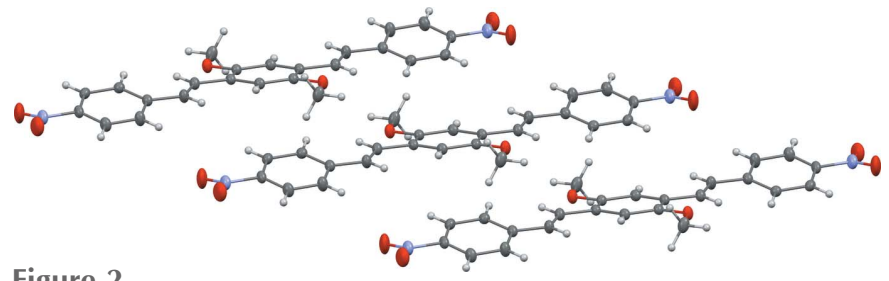

Figure 2

Ladder-like stack of DBDB molecules; the distance between the mean planes of the central phenyl rings within the stack is 3.451 (1) $\AA$.
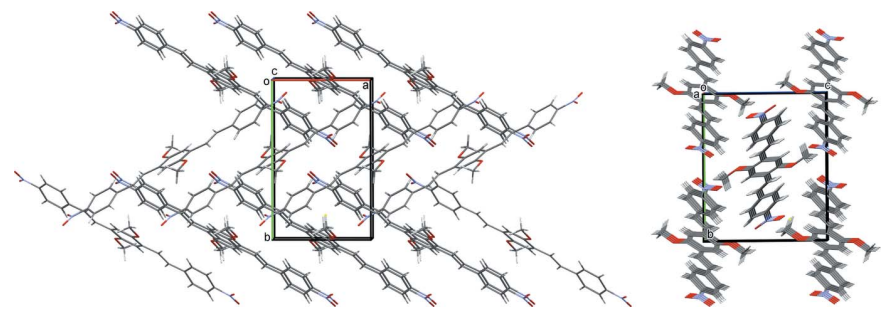

Figure 3

The packing in the crystal of the title compound.

\section{Database survey}

A search of the Cambridge Crystallographic Database (CSD version 5.40, update of September 2019; Groom et al., 2016) for the title molecule returned no results. Two entries for compounds with the same core and unsubstituted terminal rings were found. Over 30 entries were found for variously substituted molecules with the same core, of which 10 entries correspond to para-substituted terminal aromatic groups. Among them halogen-substituted molecules [refcodes: VIQCAB (Xu et al., 2013), ODOHOG (Sun et al., 2013), ODOJAU (Sun et al., 2013)], as well as molecules with cyano (OBUHAV; Xu et al., 2013), carboxyl (TOJDEE, TOJDII; Cárdenas et al., 2019) and alkylcarboxylate (TOJCUT; Cárdenas et al., 2019) groups in the para-position have been reported. Most of the molecules demonstrate dihedral angles between the central fragment and the terminal rings ranging from 5.0 (1) to $36.1(1)^{\circ}$. One notable exception is the chlorine-substituted compound (VIQCAB; Xu et al., 2013), for which the angles between central and the terminal aromatic rings are $43.82(16)$ and $67.38(17)^{\circ}$.

\section{Optical studies in solution}

A solution of the title compound in dioxane (at $10 \mathrm{mM}$ concentration) in a quartz sample cuvette $(10 \mathrm{~mm}$ optical path length) was used for optical absorption and emission studies. All measurements were carried out at ambient temperature. The corresponding spectra are shown in Fig. 4. Peak positions,

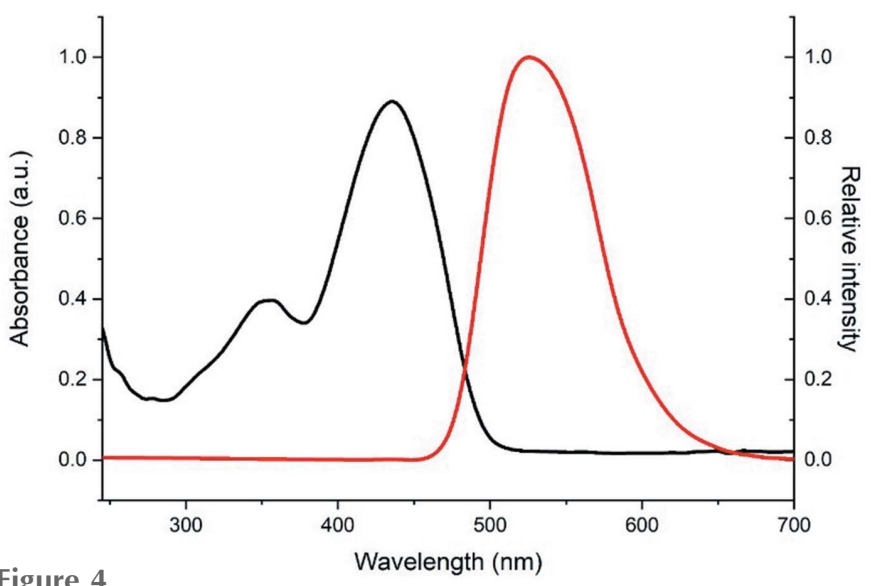

Figure 4

Normalized absorption (black) and emission (red) spectra of the title compound measured in dioxane solution. 
Table 1

Selected energy parameters (gas phase).

\begin{tabular}{lc}
\hline Total Energy $(\mathrm{eV})$ & -40479.535 \\
$E_{\text {HOMO }}(\mathrm{eV})$ & -5.813 \\
$E_{\text {LUMO }}(\mathrm{eV})$ & -3.096 \\
HOMO-LUMO gap $(\mathrm{eV})$ & 2.717 \\
$\mathrm{~S}_{0}-\mathrm{S}_{1}$ vertical excitation $(\mathrm{nm})$ & 497.44 \\
$\mathrm{~S}_{1}-\mathrm{S}_{0}$ vertical emission $(\mathrm{nm})$ & 546.03 \\
\hline
\end{tabular}

Table 2

Selected X-ray and DFT ground-state geometry parameter $\left(\AA,{ }^{\circ}\right)$ comparison.

\begin{tabular}{lll}
\hline Bonds/angles & Experimental & Calculated \\
\hline O1-C1 & $1.3663(14)$ & 1.3652 \\
O1-C12 & $1.4248(15)$ & 1.4207 \\
O2-N1 & $1.2321(16)$ & 1.2253 \\
N1-C9 & $1.4665(16)$ & 1.4723 \\
& & \\
C5-C4-C3 & $127.07(11)$ & 126.76 \\
C4-C5-C6 & $125.13(11)$ & 126.38 \\
C1-O1-C12 & $117.68(9)$ & 119.05 \\
C8-C9-N1 & $118.92(11)$ & 119.26 \\
\hline
\end{tabular}

as well as band shapes are in good agreement with those previously reported (Nakaya et al., 1966). Fluorescence was measured at the excitation wavelength of $434 \mathrm{~nm}$, chosen from the absorption spectrum, and had a maximum at $525 \mathrm{~nm}$. The $\mathrm{E}_{0-0}$ transition energy was estimated to be at $483 \mathrm{~nm}$ $(2.57 \mathrm{eV})$.

\section{DFT calculations}

In an effort to further elucidate the nature of the electronic radiative transitions in the title compound, DFT and timedependent (TD) DFT calculations were carried out with GAUSSIAN 16 software (Frisch et al., 2016). The standard B3LYP functional with the 6-311G(d,p) basis set was used to optimize both the ground and first excited states of the title molecule and to obtain vertical excitation and emission energies, HOMO ( $\left.E_{\mathrm{HOMO}}\right)$ and LUMO $\left(E_{\mathrm{LUMO}}\right)$ energies and their difference (Fig. 5). All of the calculated parameters are for the gas phase of the title compound. Both optimized geometries were confirmed to be the true minima via vibrational frequency analysis. The summary of calculated energy parameters is presented in Table 1 . The calculated geometry parameters (bond lengths and angles) are in good agreement with the experimental data (Table 2).
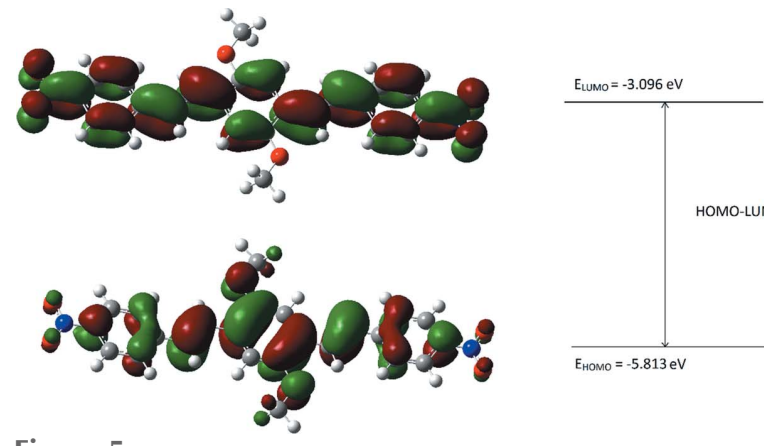

Figure 5

HOMO and LUMO orbitals with corresponding energy values and gap.
Table 3

Experimental details.

\begin{tabular}{|c|c|}
\hline \multicolumn{2}{|l|}{ Crystal data } \\
\hline Chemical formula & $\mathrm{C}_{24} \mathrm{H}_{20} \mathrm{~N}_{2} \mathrm{O}_{6}$ \\
\hline$M_{\mathrm{r}}$ & 432.42 \\
\hline Crystal system, space group & Monoclinic, $P 2_{1} / n$ \\
\hline Temperature $(\mathrm{K})$ & 100 \\
\hline$a, b, c(\AA)$ & $\begin{array}{l}7.9074(10), 12.4794(16), \\
10.6248(14)\end{array}$ \\
\hline$\beta\left({ }^{\circ}\right)$ & $102.394(3)$ \\
\hline$V\left(\AA^{6}\right)$ & $1024.0(2)$ \\
\hline$Z$ & 2 \\
\hline Radiation type & Мо $K \alpha$ \\
\hline$\mu\left(\mathrm{mm}^{-1}\right)$ & 0.10 \\
\hline Crystal size $(\mathrm{mm})$ & $0.22 \times 0.15 \times 0.11$ \\
\hline \multicolumn{2}{|l|}{ Data collection } \\
\hline Diffractometer & Bruker APEXII CCD \\
\hline Absorption correction & $\begin{array}{l}\text { Multi-scan (SADABS; Bruker, } \\
\text { 2016) }\end{array}$ \\
\hline$T_{\min }, T_{\max }$ & $0.653,0.746$ \\
\hline $\begin{array}{l}\text { No. of measured, independent and } \\
\text { observed }[I>2 \sigma(I)] \text { reflections }\end{array}$ & $32090,3460,2542$ \\
\hline$R_{\text {int }}$ & 0.055 \\
\hline$(\sin \theta / \lambda)_{\max }\left(\AA^{-1}\right)$ & 0.738 \\
\hline \multicolumn{2}{|l|}{ Refinement } \\
\hline$R\left[F^{2}>2 \sigma\left(F^{2}\right)\right], w R\left(F^{2}\right), S$ & $0.048,0.145,1.04$ \\
\hline No. of reflections & 3460 \\
\hline No. of parameters & 146 \\
\hline H-atom treatment & $\mathrm{H}$-atom parameters constrained \\
\hline$\Delta \rho_{\max }, \Delta \rho_{\min }\left(\mathrm{e} \AA^{-3}\right)$ & $0.49,-0.21$ \\
\hline
\end{tabular}

Computer programs: APEX3 (Bruker, 2016), SAINT (Bruker, 2016), SHELXT2017/1 (Sheldrick, 2015a), SHELXL2017/1 (Sheldrick, 2015b), OLEX2 (Dolomanov et al., 2009), Mercury (Macrae et al., 2020).

\section{Synthesis and crystallization}

The synthesis of title compound was carried out as described in the literature (Nakaya et al., 1966; Caruso et al., 2005). The obtained material was recrystallized by slow evaporation of ethanol solution giving dark-red block-shaped crystals.

\section{Refinement}

Crystal data, data collection and structure refinement details are summarized in Table 3. $\mathrm{H}$ atoms were placed in calculated positions $(0.95-0.98 \AA)$ and refined as riding with $U_{\text {iso }}(\mathrm{H})=$ $1.2 U_{\text {eq }}(\mathrm{C})$ or $1.5 U_{\text {eq }}(\mathrm{C}-$ methyl $)$.

\section{Funding information}

Funding for this research was provided by: National Science Foundation (grant No. DMR-0934212 ; grant No. DMR1523611); Foundation for the National Institutes of Health (grant No. 1R21NS084353-01).

\section{References}

Bruker (2016). APEX3, SAINT and SADABS. Bruker AXS Inc., Madison, Wisconsin, USA.

Cárdenas, J. C., Aguirre-Díaz, L. M., Galindo, J. F., Alí-Torres, J., Ochoa-Puentes, C., Echeverri, M., Gómez-Lor, B., Monge, M. Á., Gutiérrez-Puebla, E. \& Sierra, C. A. (2019). Cryst. Growth Des. 19, 3913-3922. 
Caruso, U., Casalboni, M., Fort, A., Fusco, M., Panunzi, B., Quatela, A., Roviello, A. \& Sarcinelli, F. (2005). Opt. Mater. 27, 1800-1810. Dolomanov, O. V., Bourhis, L. J., Gildea, R. J., Howard, J. A. K. \& Puschmann, H. (2009). J. Appl. Cryst. 42, 339-341.

Frisch, M. J., Trucks, G. W., Schlegel, H. B., Scuseria, G. E., Robb, M. A., Cheeseman, J. R., Scalmani, G., Barone, V., Petersson, G. A., Nakatsuji, H., Li, X., Caricato, M., Marenich, A. V., Bloino, J., Janesko, B. G., Gomperts, R., Mennucci, B., Hratchian, H. P., Ortiz, J. V., Izmaylov, A. F., Sonnenberg, J. L., Williams-Young, D., Ding, F., Lipparini, F., Egidi, F., Goings, J., Peng, B., Petrone, A., Henderson, T., Ranasinghe, D., Zakrzewski, V. G., Gao, J., Rega, N., Zheng, G., Liang, W., Hada, M., Ehara, M., Toyota, K., Fukuda, R., Hasegawa, J., Ishida, M., Nakajima, T., Honda, Y., Kitao, O., Nakai, H., Vreven, T., Throssell, K., Montgomery, J. A. Jr, Peralta, J. E., Ogliaro, F., Bearpark, M. J., Heyd, J. J., Brothers, E. N., Kudin, K. N., Staroverov, V. N., Keith, T. A., Kobayashi, R., Normand, J., Raghavachari, K., Rendell, A. P., Burant, J. C., Iyengar, S. S., Tomasi, J., Cossi, M., Millam, J. M., Klene, M., Adamo, C., Cammi,
R., Ochterski, J. W., Martin, R. L., Morokuma, K., Farkas, O., Foresman, J. B. \& Fox, D. J. (2016). GAUSSIAN16. Revision C. 01 Gaussian Inc., Wallingford, CT, USA. http://www.gaussian.com.

Groom, C. R., Bruno, I. J., Lightfoot, M. P. \& Ward, S. C. (2016). Acta Cryst. B72, 171-179.

He, G. S., Tan, L.-S., Zheng, Q. \& Prasad, P. N. (2008). Chem. Rev. 108, $1245-1330$

Macrae, C. F., Sovago, I., Cottrell, S. J., Galek, P. T. A., McCabe, P., Pidcock, E., Platings, M., Shields, G. P., Stevens, J. S., Towler, M. \& Wood, P. A. (2020). J. Appl. Cryst. 53, 226-235.

Nakaya, T. \& Imoto, M. (1966). Bull. Chem. Soc. Jpn, 39, 15471551.

Sheldrick, G. M. (2015a). Acta Cryst. A71, 3-8.

Sheldrick, G. M. (2015b). Acta Cryst. C71, 3-8.

Sun, C.-L., Li, J., Geng, H.-W., Li, H., Ai, Y., Wang, Q., Pan, S.-L. \& Zhang, H.-L. (2013). Chem. Asian J. 8, 3091-3100.

Xu, Z., Liao, Q., Shi, X., Li, H., Zhang, H. \& Fu, H. (2013). J. Mater. Chem. B, 1, 6035-6041. 


\section{supporting information}

Acta Cryst. (2020). E76, 940-943 [https://doi.org/10.1107/S205698902000674X]

Molecular and crystal structure, optical properties and DFT studies of 1,4-dimethoxy-2,5-bis[2-(4-nitrophenyl)ethenyl]benzene

Georgii Bogdanov, Evgenii Oskolkov, Jenna Bustos, Viktor Glebov, John P. Tillotson and Tatiana V. Timofeeva

Computing details

Data collection: APEX3 (Bruker, 2016); cell refinement: SAINT (Bruker, 2016); data reduction: SAINT (Bruker, 2016); program(s) used to solve structure: SHELXT2017/1 (Sheldrick, 2015a); program(s) used to refine structure:

SHELXL2017/1 (Sheldrick, 2015b), OLEX2 (Dolomanov et al., 2009); molecular graphics: Mercury (Macrae et al., 2020); software used to prepare material for publication: Mercury (Macrae et al., 2020).

1,4-Dimethoxy-2,5-bis[2-(4-nitrophenyl)ethenyl]benzene

Crystal data

$\mathrm{C}_{24} \mathrm{H}_{20} \mathrm{~N}_{2} \mathrm{O}_{6}$

$M_{r}=432.42$

Monoclinic, $P 2{ }_{1} / n$

$a=7.9074(10) \AA$

$b=12.4794(16) \AA$

$c=10.6248(14) \AA$

$\beta=102.394(3)^{\circ}$

$V=1024.0(2) \AA^{3}$

$Z=2$

\section{Data collection}

Bruker APEXII CCD

diffractometer

$\varphi$ and $\omega$ scans

Absorption correction: multi-scan

(SADABS; Bruker, 2016)

$T_{\text {min }}=0.653, T_{\max }=0.746$

32090 measured reflections

\section{Refinement}

Refinement on $F^{2}$

Least-squares matrix: full

$R\left[F^{2}>2 \sigma\left(F^{2}\right)\right]=0.048$

$w R\left(F^{2}\right)=0.145$

$S=1.03$

3460 reflections

146 parameters

0 restraints
$F(000)=452$

$D_{\mathrm{x}}=1.403 \mathrm{Mg} \mathrm{m}^{-3}$

Mo $K \alpha$ radiation, $\lambda=0.71073 \AA$

Cell parameters from 6481 reflections

$\theta=2.6-31.3^{\circ}$

$\mu=0.10 \mathrm{~mm}^{-1}$

$T=100 \mathrm{~K}$

Block, red

$0.22 \times 0.15 \times 0.11 \mathrm{~mm}$

3460 independent reflections

2542 reflections with $I>2 \sigma(I)$

$R_{\text {int }}=0.055$

$\theta_{\text {max }}=31.7^{\circ}, \theta_{\text {min }}=2.6^{\circ}$

$h=-11 \rightarrow 11$

$k=-18 \rightarrow 18$

$l=-15 \rightarrow 15$

Primary atom site location: dual

Secondary atom site location: difference Fourier map

Hydrogen site location: inferred from neighbouring sites

$\mathrm{H}$-atom parameters constrained

$w=1 /\left[\sigma^{2}\left(F_{\mathrm{o}}^{2}\right)+(0.0723 P)^{2}+0.3749 P\right]$

where $P=\left(F_{\mathrm{o}}{ }^{2}+2 F_{\mathrm{c}}{ }^{2}\right) / 3$ 
$(\Delta / \sigma)_{\max }<0.001$

$\Delta \rho_{\max }=0.49 \mathrm{e} \AA^{-3}$

$\Delta \rho_{\min }=-0.21$ e $\AA^{-3}$

Special details

Geometry. All esds (except the esd in the dihedral angle between two 1.s. planes) are estimated using the full covariance matrix. The cell esds are taken into account individually in the estimation of esds in distances, angles and torsion angles; correlations between esds in cell parameters are only used when they are defined by crystal symmetry. An approximate (isotropic) treatment of cell esds is used for estimating esds involving l.s. planes.

Fractional atomic coordinates and isotropic or equivalent isotropic displacement parameters $\left(\AA^{2}\right)$

\begin{tabular}{|c|c|c|c|c|}
\hline & $x$ & $y$ & $z$ & $U_{\text {iso }} * / U_{\text {eq }}$ \\
\hline $\mathrm{O} 1$ & $1.12432(11)$ & $0.50902(7)$ & $0.27624(8)$ & $0.0223(2)$ \\
\hline $\mathrm{O} 2$ & $-0.13219(13)$ & $0.91355(10)$ & $0.42105(12)$ & $0.0423(3)$ \\
\hline $\mathrm{O} 3$ & $-0.08612(15)$ & $0.85312(11)$ & $0.61675(12)$ & $0.0453(3)$ \\
\hline N1 & $-0.04597(14)$ & $0.86252(10)$ & $0.51181(12)$ & $0.0301(3)$ \\
\hline $\mathrm{C} 1$ & $1.05803(14)$ & $0.50680(9)$ & $0.38485(11)$ & $0.0176(2)$ \\
\hline $\mathrm{C} 2$ & $0.90285(14)$ & $0.55533(9)$ & $0.39523(11)$ & $0.0185(2)$ \\
\hline $\mathrm{H} 2$ & 0.836944 & 0.592801 & 0.323366 & $0.022 *$ \\
\hline $\mathrm{C} 3$ & $0.84235(14)$ & $0.54988(9)$ & $0.50948(11)$ & $0.0178(2)$ \\
\hline $\mathrm{C} 10$ & $0.18811(15)$ & $0.73214(10)$ & $0.58176(12)$ & $0.0215(2)$ \\
\hline $\mathrm{H} 10$ & 0.137655 & 0.713154 & 0.652137 & $0.026^{*}$ \\
\hline $\mathrm{C} 4$ & $0.67887(14)$ & $0.59782(10)$ & $0.52387(11)$ & $0.0202(2)$ \\
\hline $\mathrm{H} 4$ & 0.640701 & 0.580352 & 0.600263 & $0.024^{*}$ \\
\hline C6 & $0.41503(15)$ & $0.71106(10)$ & $0.46101(11)$ & $0.0196(2)$ \\
\hline C11 & $0.33818(15)$ & $0.68241(9)$ & $0.56370(11)$ & $0.0198(2)$ \\
\hline H11 & 0.389852 & 0.627975 & 0.621909 & $0.024 *$ \\
\hline $\mathrm{C} 9$ & $0.11358(14)$ & $0.81049(10)$ & $0.49411(12)$ & $0.0217(2)$ \\
\hline $\mathrm{C} 5$ & $0.57748(16)$ & $0.66340(10)$ & $0.44141(12)$ & $0.0225(2)$ \\
\hline H5 & 0.613011 & 0.680685 & 0.363923 & $0.027 *$ \\
\hline $\mathrm{C} 8$ & $0.18426(16)$ & $0.84114(11)$ & $0.39171(12)$ & 0.0249 \\
\hline H8 & 0.131401 & 0.895451 & 0.333775 & $0.030^{*}$ \\
\hline $\mathrm{C} 7$ & $0.33514(17)$ & $0.79047(11)$ & $0.37539(12)$ & $0.0252(3)$ \\
\hline H7 & 0.384749 & 0.810152 & 0.304835 & $0.030^{*}$ \\
\hline $\mathrm{C} 12$ & $1.03435(16)$ & $0.57061(12)$ & $0.16982(12)$ & $0.0269(3)$ \\
\hline $\mathrm{H} 12 \mathrm{~A}$ & 1.097995 & 0.568337 & 0.100234 & $0.040^{*}$ \\
\hline H12B & 1.025026 & 0.645017 & 0.197031 & $0.040^{*}$ \\
\hline $\mathrm{H} 12 \mathrm{C}$ & 0.918153 & 0.540834 & 0.138992 & $0.040^{*}$ \\
\hline
\end{tabular}

Atomic displacement parameters $\left(\AA^{2}\right)$

\begin{tabular}{lllllll}
\hline & $U^{11}$ & $U^{22}$ & $U^{33}$ & $U^{12}$ & $U^{13}$ & $U^{23}$ \\
\hline O1 & $0.0212(4)$ & $0.0287(5)$ & $0.0177(4)$ & $0.0069(3)$ & $0.0058(3)$ & $0.0033(3)$ \\
O2 & $0.0253(5)$ & $0.0519(7)$ & $0.0485(7)$ & $0.0170(5)$ & $0.0054(5)$ & $0.0069(5)$ \\
O3 & $0.0363(6)$ & $0.0580(8)$ & $0.0491(7)$ & $0.0163(5)$ & $0.0260(5)$ & $0.0067(6)$ \\
N1 & $0.0192(5)$ & $0.0331(6)$ & $0.0387(6)$ & $0.0057(4)$ & $0.0082(4)$ & $-0.0013(5)$ \\
C1 & $0.0175(5)$ & $0.0183(5)$ & $0.0172(5)$ & $0.0007(4)$ & $0.0040(4)$ & $-0.0014(4)$ \\
C2 & $0.0168(5)$ & $0.0200(5)$ & $0.0180(5)$ & $0.0027(4)$ & $0.0018(4)$ & $0.0010(4)$ \\
C3 & $0.0152(5)$ & $0.0181(5)$ & $0.0198(5)$ & $0.0018(4)$ & $0.0029(4)$ & $-0.0014(4)$
\end{tabular}




\begin{tabular}{lllllll} 
C10 & $0.0191(5)$ & $0.0228(6)$ & $0.0237(5)$ & $-0.0025(4)$ & $0.0071(4)$ & $-0.0014(4)$ \\
C4 & $0.0180(5)$ & $0.0234(5)$ & $0.0195(5)$ & $0.0026(4)$ & $0.0049(4)$ & $-0.0007(4)$ \\
C6 & $0.0167(5)$ & $0.0211(5)$ & $0.0206(5)$ & $0.0023(4)$ & $0.0033(4)$ & $-0.0012(4)$ \\
C11 & $0.0180(5)$ & $0.0199(5)$ & $0.0212(5)$ & $0.0000(4)$ & $0.0034(4)$ & $0.0003(4)$ \\
C9 & $0.0148(5)$ & $0.0246(6)$ & $0.0257(6)$ & $0.0028(4)$ & $0.0045(4)$ & $-0.0043(4)$ \\
C5 & $0.0211(5)$ & $0.0250(6)$ & $0.0235(6)$ & $0.0059(4)$ & $0.0092(4)$ & $0.0036(4)$ \\
C8 & $0.0227(6)$ & $0.0284(6)$ & $0.0235(6)$ & $0.0083(5)$ & $0.0044(4)$ & $0.0030(5)$ \\
C7 & $0.0247(6)$ & $0.0301(6)$ & $0.0225(6)$ & $0.0085(5)$ & $0.0091(5)$ & $0.0052(5)$ \\
C12 & $0.0212(5)$ & $0.0388(7)$ & $0.0211(6)$ & $0.0048(5)$ & $0.0055(4)$ & $0.0086(5)$ \\
\hline
\end{tabular}

Geometric parameters $\left(\AA,{ }^{o}\right)$

\begin{tabular}{|c|c|c|c|}
\hline $\mathrm{O} 1-\mathrm{C} 1$ & $1.3663(14)$ & $\mathrm{C} 4-\mathrm{C} 5$ & $1.3337(16)$ \\
\hline $\mathrm{O} 1-\mathrm{C} 12$ & $1.4248(15)$ & $\mathrm{C} 6-\mathrm{C} 11$ & $1.4043(16)$ \\
\hline $\mathrm{O} 2-\mathrm{N} 1$ & $1.2321(16)$ & $\mathrm{C} 6-\mathrm{C} 5$ & $1.4704(16)$ \\
\hline $\mathrm{O} 3-\mathrm{N} 1$ & $1.2287(16)$ & $\mathrm{C} 6-\mathrm{C} 7$ & 1.4007 (17) \\
\hline $\mathrm{N} 1-\mathrm{C} 9$ & $1.4665(16)$ & $\mathrm{C} 11-\mathrm{H} 11$ & 0.9500 \\
\hline $\mathrm{C} 1-\mathrm{C} 2$ & $1.3938(15)$ & $\mathrm{C} 9-\mathrm{C} 8$ & $1.3796(18)$ \\
\hline $\mathrm{C} 1-\mathrm{C} 3^{\mathrm{i}}$ & $1.4148(16)$ & $\mathrm{C} 5-\mathrm{H} 5$ & 0.9500 \\
\hline $\mathrm{C} 2-\mathrm{H} 2$ & 0.9500 & $\mathrm{C} 8-\mathrm{H} 8$ & 0.9500 \\
\hline $\mathrm{C} 2-\mathrm{C} 3$ & $1.3986(16)$ & $\mathrm{C} 8-\mathrm{C} 7$ & $1.3936(17)$ \\
\hline $\mathrm{C} 3-\mathrm{C} 4$ & $1.4619(15)$ & $\mathrm{C} 7-\mathrm{H} 7$ & 0.9500 \\
\hline $\mathrm{C} 10-\mathrm{H} 10$ & 0.9500 & $\mathrm{C} 12-\mathrm{H} 12 \mathrm{~A}$ & 0.9800 \\
\hline $\mathrm{C} 10-\mathrm{C} 11$ & $1.3885(16)$ & $\mathrm{C} 12-\mathrm{H} 12 \mathrm{~B}$ & 0.9800 \\
\hline $\mathrm{C} 10-\mathrm{C} 9$ & $1.3910(17)$ & $\mathrm{C} 12-\mathrm{H} 12 \mathrm{C}$ & 0.9800 \\
\hline $\mathrm{C} 4-\mathrm{H} 4$ & 0.9500 & & \\
\hline $\mathrm{C} 1-\mathrm{O} 1-\mathrm{C} 12$ & $117.68(9)$ & $\mathrm{C} 10-\mathrm{C} 11-\mathrm{C} 6$ & $121.25(11)$ \\
\hline $\mathrm{O} 2-\mathrm{N} 1-\mathrm{C} 9$ & $118.37(12)$ & $\mathrm{C} 10-\mathrm{C} 11-\mathrm{H} 11$ & 119.4 \\
\hline $\mathrm{O} 3-\mathrm{N} 1-\mathrm{O} 2$ & $123.52(12)$ & $\mathrm{C} 6-\mathrm{C} 11-\mathrm{H} 11$ & 119.4 \\
\hline $\mathrm{O} 3-\mathrm{N} 1-\mathrm{C} 9$ & $118.11(11)$ & $\mathrm{C} 10-\mathrm{C} 9-\mathrm{N} 1$ & $118.55(11)$ \\
\hline $\mathrm{O} 1-\mathrm{C} 1-\mathrm{C} 2$ & $124.19(10)$ & $\mathrm{C} 8-\mathrm{C} 9-\mathrm{N} 1$ & $118.92(11)$ \\
\hline $\mathrm{O} 1-\mathrm{C} 1-\mathrm{C} 3^{\mathrm{i}}$ & $115.54(10)$ & $\mathrm{C} 8-\mathrm{C} 9-\mathrm{C} 10$ & $122.53(11)$ \\
\hline $\mathrm{C} 2-\mathrm{C} 1-\mathrm{C} 3^{\mathrm{i}}$ & $120.27(10)$ & $\mathrm{C} 4-\mathrm{C} 5-\mathrm{C} 6$ & $125.13(11)$ \\
\hline $\mathrm{C} 1-\mathrm{C} 2-\mathrm{H} 2$ & 119.4 & $\mathrm{C} 4-\mathrm{C} 5-\mathrm{H} 5$ & 117.4 \\
\hline $\mathrm{C} 1-\mathrm{C} 2-\mathrm{C} 3$ & $121.23(10)$ & $\mathrm{C} 6-\mathrm{C} 5-\mathrm{H} 5$ & 117.4 \\
\hline $\mathrm{C} 3-\mathrm{C} 2-\mathrm{H} 2$ & 119.4 & $\mathrm{C} 9-\mathrm{C} 8-\mathrm{H} 8$ & 120.8 \\
\hline $\mathrm{C} 1-\mathrm{C} 3-\mathrm{C} 4$ & $118.41(10)$ & $\mathrm{C} 9-\mathrm{C} 8-\mathrm{C} 7$ & $118.35(11)$ \\
\hline $\mathrm{C} 2-\mathrm{C} 3-\mathrm{C}^{\mathrm{i}}$ & $118.50(10)$ & $\mathrm{C} 7-\mathrm{C} 8-\mathrm{H} 8$ & 120.8 \\
\hline $\mathrm{C} 2-\mathrm{C} 3-\mathrm{C} 4$ & $123.08(10)$ & $\mathrm{C} 6-\mathrm{C} 7-\mathrm{H} 7$ & 119.4 \\
\hline $\mathrm{C} 11-\mathrm{C} 10-\mathrm{H} 10$ & 120.9 & $\mathrm{C} 8-\mathrm{C} 7-\mathrm{C} 6$ & $121.27(11)$ \\
\hline $\mathrm{C} 11-\mathrm{C} 10-\mathrm{C} 9$ & $118.26(11)$ & $\mathrm{C} 8-\mathrm{C} 7-\mathrm{H} 7$ & 119.4 \\
\hline $\mathrm{C} 9-\mathrm{C} 10-\mathrm{H} 10$ & 120.9 & $\mathrm{O} 1-\mathrm{C} 12-\mathrm{H} 12 \mathrm{~A}$ & 109.5 \\
\hline $\mathrm{C} 3-\mathrm{C} 4-\mathrm{H} 4$ & 116.5 & $\mathrm{O} 1-\mathrm{C} 12-\mathrm{H} 12 \mathrm{~B}$ & 109.5 \\
\hline $\mathrm{C} 5-\mathrm{C} 4-\mathrm{C} 3$ & $127.07(11)$ & $\mathrm{O} 1-\mathrm{C} 12-\mathrm{H} 12 \mathrm{C}$ & 109.5 \\
\hline $\mathrm{C} 5-\mathrm{C} 4-\mathrm{H} 4$ & 116.5 & $\mathrm{H} 12 \mathrm{~A}-\mathrm{C} 12-\mathrm{H} 12 \mathrm{~B}$ & 109.5 \\
\hline $\mathrm{C} 11-\mathrm{C} 6-\mathrm{C} 5$ & $122.95(11)$ & $\mathrm{H} 12 \mathrm{~A}-\mathrm{C} 12-\mathrm{H} 12 \mathrm{C}$ & 109.5 \\
\hline $\mathrm{C} 7-\mathrm{C} 6-\mathrm{C} 11$ & $118.34(10)$ & $\mathrm{H} 12 \mathrm{~B}-\mathrm{C} 12-\mathrm{H} 12 \mathrm{C}$ & 109.5 \\
\hline
\end{tabular}


$\mathrm{C} 7-\mathrm{C} 6-\mathrm{C} 5$

$\mathrm{O} 1-\mathrm{C} 1-\mathrm{C} 2-\mathrm{C} 3$

$\mathrm{O} 2-\mathrm{N} 1-\mathrm{C} 9-\mathrm{C} 10$

$\mathrm{O} 2-\mathrm{N} 1-\mathrm{C} 9-\mathrm{C} 8$

$\mathrm{O} 3-\mathrm{N} 1-\mathrm{C} 9-\mathrm{C} 10$

$\mathrm{O} 3-\mathrm{N} 1-\mathrm{C} 9-\mathrm{C} 8$

$\mathrm{N} 1-\mathrm{C} 9-\mathrm{C} 8-\mathrm{C} 7$

$\mathrm{C} 1-\mathrm{C} 2-\mathrm{C} 3-\mathrm{C}^{\mathrm{i}}$

$\mathrm{C} 1-\mathrm{C} 2-\mathrm{C} 3-\mathrm{C} 4$

$\mathrm{C} 1-\mathrm{C} 3-\mathrm{C} 4-\mathrm{C} 5$

$\mathrm{C} 2-\mathrm{C} 3-\mathrm{C} 4-\mathrm{C} 5$

$\mathrm{C} 3-\mathrm{C} 1-\mathrm{C} 2-\mathrm{C} 3$

$\mathrm{C} 3-\mathrm{C} 4-\mathrm{C} 5-\mathrm{C} 6$

$\mathrm{C} 10-\mathrm{C} 9-\mathrm{C} 8-\mathrm{C} 7$
$118.69(11)$

$$
\begin{aligned}
& 179.95(11) \\
& -164.27(12) \\
& 15.92(19) \\
& 15.98(19) \\
& -163.83(13) \\
& -179.49(12) \\
& -0.34(18) \\
& -178.81(11) \\
& 172.08(12) \\
& -9.4(2) \\
& 0.34(19) \\
& -179.01(12) \\
& 0.7(2)
\end{aligned}
$$

$$
\begin{aligned}
& 179.35(11) \\
& -0.84(19) \\
& -7.6(2) \\
& 0.74(19) \\
& 0.93(18) \\
& -0.6(2) \\
& 177.54(11) \\
& -177.76(12) \\
& -0.89(18) \\
& 170.84(13) \\
& 4.15(17) \\
& -176.23(11)
\end{aligned}
$$

Symmetry code: (i) $-x+2,-y+1,-z+1$. 